\title{
Isolated posterior uveal effusion: expanding the spectrum of the uveal effusion syndrome
}

This article was published in the following Dove Press journal:

Clinical Ophthalmology

30 December 2014

Number of times this article has been viewed

\section{Scott E Pautler' \\ David J Browning ${ }^{2}$}

'Department of Ophthalmology, University of South Florida, Morsani College of Medicine, Tampa, FL, USA ${ }^{2}$ Charlotte Ear Eye Nose and Throat Associates, Charlotte, NC, USA
Correspondence: Scott E Pautler Retina Vitreous Associates of Florida, 2705 W St Isabel Street, Tampa,

FL 33607, USA

Tel + I 8138795795

Fax + I 8138774578

Email pautlers@aol.com
Abstract: Uveal effusion syndrome usually causes peripheral chorioretinal detachment, but posterior effusion may present as isolated macular edema with serous macular detachment in the setting of hyperopia and a thickened posterior choroid. Carbonic anhydrase inhibitors may be effective to treat this condition.

Keywords: uveal effusion, serous, macular detachment, macular edema

\section{Introduction}

The uveal effusion syndrome (UES) usually occurs in hyperopic eyes with abnormally thick scleras and choroids. The thickened sclera impairs diffusion of fluid from the suprachoroidal space. It is speculated that compression of the vortex veins may increase the hydrostatic pressure within the choroid and thereby promote fluid transudation, which is worsened if the choroidal vessel walls are hyperpermeable. As a result of one or more factors, serous choroidal detachment and serous retinal detachment may occur. $^{1}$

In this report, an unusual case and its treatment are documented: bilateral posterior uveal effusion without peripheral choroidal effusion was treated successfully with oral and topical carbonic anhydrase inhibitors.

\section{Case report}

A 73-year-old white male was seen for blurred vision attributed to neovascular age-related maculopathy that did not respond to intravitreal bevacizumab and triamcinolone. He reported good vision from birth and began wearing +7 diopter hyperopic correction lenses as a young adult. He had no past ocular trauma, inflammation, or known ocular disease other than narrow angles, for which he had remotely received laser iridotomy. He had medically controlled hypertension and hyperlipidemia. $\mathrm{He}$ denied headache or niacin use, and otherwise had a negative review of systems. Several months earlier, he had developed blurred vision attributed to macular edema (Figure 1A and B) and cataract. He underwent uneventful cataract surgery in both eyes; however, his blurred vision persisted in the right eye, and the macular edema increased bilaterally. Treatment with intravitreal bevacizumab twice and with intravitreal triamcinolone did not improve the condition.

At the time of the initial examination with one of the authors (SEP), the patient's visual acuity was 20/32 OD (oculus dexter, right eye) and 20/20 OS (oculus sinister, left eye). OD, there was mild distortion on the Amsler grid. The intraocular pressure was $12 \mathrm{mmHg}$ OD and $15 \mathrm{mmHg}$ OS. The anterior segments were normal; they had patent peripheral iridotomies and horizontal corneal diameters of $12 \mathrm{~mm}$. The posterior 

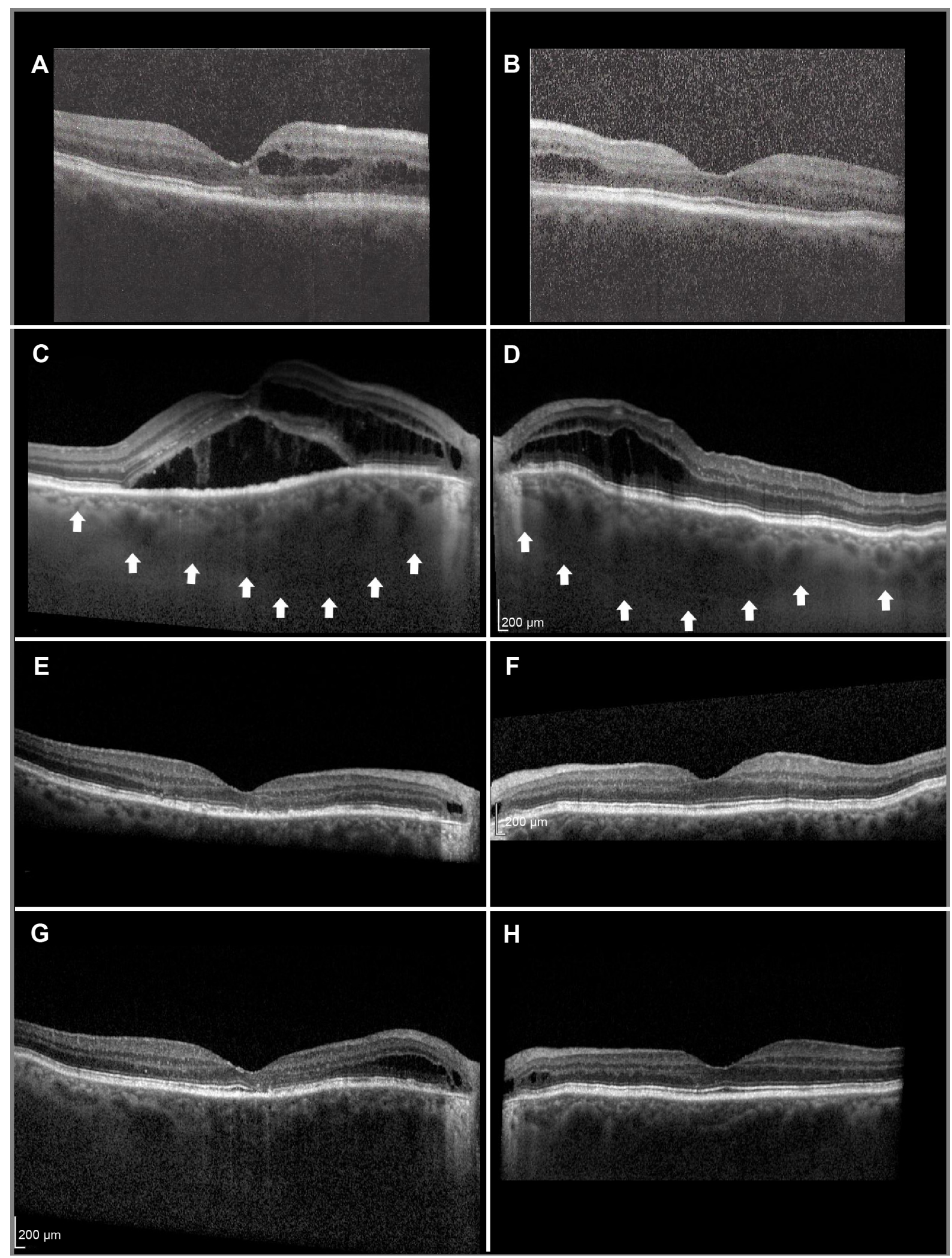

$\mathbf{F}$

\section{if}

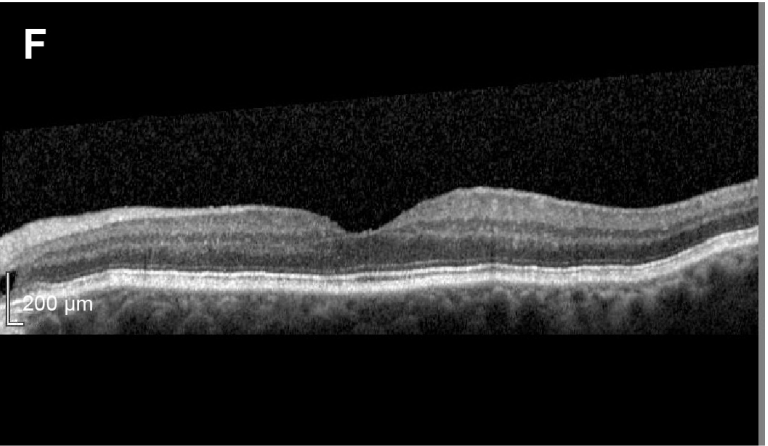

H

Figure I Serial OCT scans, right eye in left column and left eye in right column.

Notes: Outer cystic retinal edema prior to cataract extraction (A and B). Increased cystic edema OU and SRF OD after cataract extraction; choroidal thickness demarcated by white arrows ( $\mathbf{C}$ and $\mathbf{D})$. Resolved edema and SRF after starting acetazolamide (E and $\mathbf{F})$. Mild return of edema at temporal disc border when carbonic anhydrase inhibitors were stopped $(\mathbf{G}$ and $\mathbf{H})$.

Abbreviations: OCT, optical coherence tomography; OU, oculus uterque (both eyes); SRF, subretinal fluid; OD, oculus dexter (right eye). 
chamber lens implants were in proper position, and there was no anterior segment inflammation. In the Schlemm's canal, there was no blood that could be seen by gonioscopy. The vitreous was free of cells. The discs were free of pit, tilt, and coloboma (Figure 2A and B). No peripapillary choroidal excavation was present. Macular edema extended from the disc into the macula and was greater in the right eye than in the left. No dome maculopathy was present. None of the following conditions were detected by ophthalmoscopy or B-scan echography: peripheral retinoschisis; retinal detachment; leopard-spot pigmentation; choroidal detachment; or posterior scleritis. Seen by the use of B-scan echography, the choroidal-scleral thickness was consistent with hyperopia. The axial lengths were $22.3 \mathrm{~mm}$ OD and $22.42 \mathrm{~mm}$ OS. Fluorescein angiography showed leakage from neither the retinal vascular system nor the retinal pigment epithelium (RPE), although there was staining of the temporal peripapillary crescent (Figure 2C and D). Indocyanine green angiography revealed late hypercyanescence in the posterior pole; this suggests late hyperpermeability. There was hypofluorescence of the temporal peripapillary crescents (Figure 2E and F). Enhanced-depth imaging optical coherence tomography (OCT) for both maculae demonstrated thick choroids (496 $\mu \mathrm{m}$ OD and $537 \mu \mathrm{m}$ OS) and outer retinal edema extending from the disc into the macula with subretinal fluid OD (Figure 1C and 1D). In each eye, the thickness of the choroid was greatest posteriorly. There was no evidence of shifting fluid. A neurological examination revealed an isolated left fourth cranial nerve palsy, but all of other examined factors, including cerebrospinal fluid pressure and composition, were normal.

Attempting to avoid surgical intervention and frustrated with the lack of response to intravitreal triamcinolone and bevacizumab, the patient was started on a trial of acetazolamide, $500 \mathrm{mg}$ extended-release sequels orally twice a day; subretinal fluid and edema were promptly resolved in both eyes (Figure 1E and F). Initial improvement in effusion was seen after 1 week on acetazolamide; central retinal thickness, seen by the use of OCT, decreased from 768/301 (OD/OS) to $718 / 296$. Four weeks later, central retinal thickness was 233/278 with minimal subretinal fluid OD and no edema oculus uterque (OU). After another 4 weeks, there was neither residual fluid nor edema in either eye. The visual acuity improved from 20/40 OD and 20/25 OS prior to treatment to 20/20 OU after treatment. However, the patient developed malaise and confusion, and he requested that the medication be discontinued. After 9 weeks of treatment, acetazolamide was tapered to $125 \mathrm{mg}$ daily. His vision was unchanged, but macular edema began to recur at the temporal crescent of the disc OD. Therefore, a course of $2 \%$ dorzolamide hydrochloride ophthalmic solution was started OU three times a day; this resulted in a reduction of retinal edema within 1 month and no change in vision. At this point, the acetazolamide was stopped. Over the next 5 months on dorzolamide monotherapy, a small area of cystic edema at the temporal crescent remained unchanged, and the patient's vision remained stable. At this point, he requested that the dorzolamide be stopped. Within a month, the edema recurred and threatened the macula of the right eye greater than the left (Figure $1 \mathrm{G}$ and $\mathrm{H}$ ). This change prompted restarting topical dorzolamide; the edema promptly retreated to the temporal crescent of the disc. The choroid remained thickened without definitive change throughout the course of treatment and follow up, which exceeded 1 year.

\section{Discussion}

Although uveal effusion involving the posterior pole has been described in the past, ${ }^{2}$ it was seen in association with peripheral effusion, presumably as a posterior extension of subretinal fluid. To the authors' knowledge, this is the first case of isolated posterior choroidal effusion that has been described in the literature. Harada et $\mathrm{al}^{2}$ reported a case of chronic UES with peripheral cilioretinal effusion, peripheral serous retinal detachment, and macular changes. OCT revealed a thickened submacular choroid and cystic retinal changes temporal to the disc with subretinal fluid extending into macula. The fluorescein angiogram showed chronic window defects of the RPE in the macula and around disc. Although not stated in the article, the macular edema and posterior subretinal fluid may have extended from the choroid via the optic disc margin and may not have resulted from an extension of peripheral subretinal fluid.

In posterior uveal effusion, the intraretinal and subretinal fluid seemed to arise from the choroid, which is unusually thick posteriorly. The fluid may have entered the intraretinal and subretinal space at the disc margin, and the presence of a temporal disc crescent may be an important predisposing factor. As the photoreceptors and RPE are attenuated at the temporal peripapillary crescent (Figure 3 ), serous exudation extends from the choroid into the extracellular space in the outer neurosensory retina. As intraretinal edema progresses into the fovea, the edema extends through the external limiting membrane into the subretinal space in the central macular area, where intraretinal edema is greatest (Figure 4). 

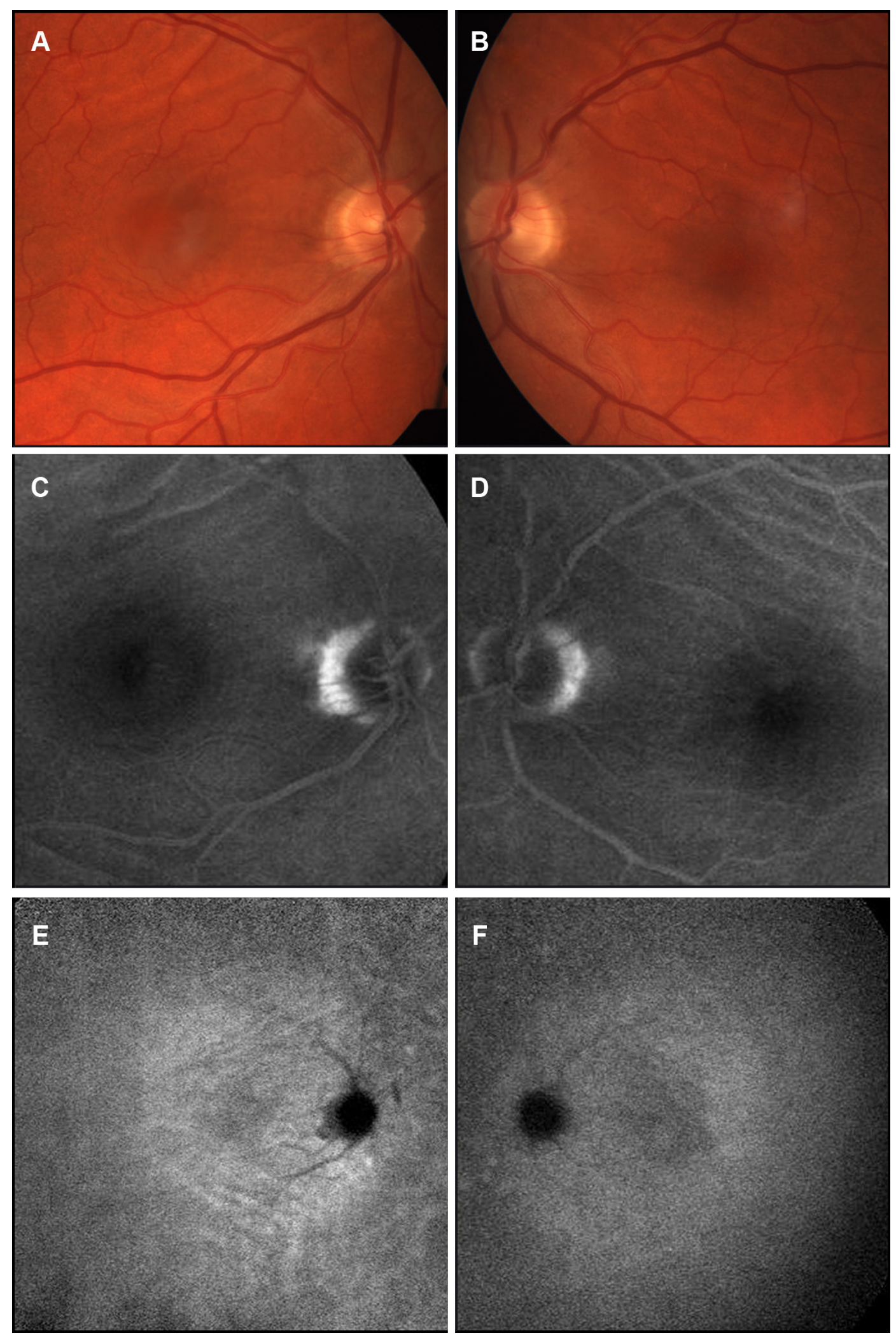

Figure 2 Composite fundus images.

Notes: Color photographs show healthy discs, temporal peripapillary crescents, and choroidal folds (A and B). Fluorescein angiograms demonstrated no macular retinal epithelial decompensation, although the crescents stained in the late phase (C and $\mathbf{D})$. Late hyperfluorescence suggests hyperpermeability in the posterior pole on an ICGA ( $\mathbf{E}$ and $\mathbf{F})$.

Abbreviation: ICGA, indocyanine green angiogram. 


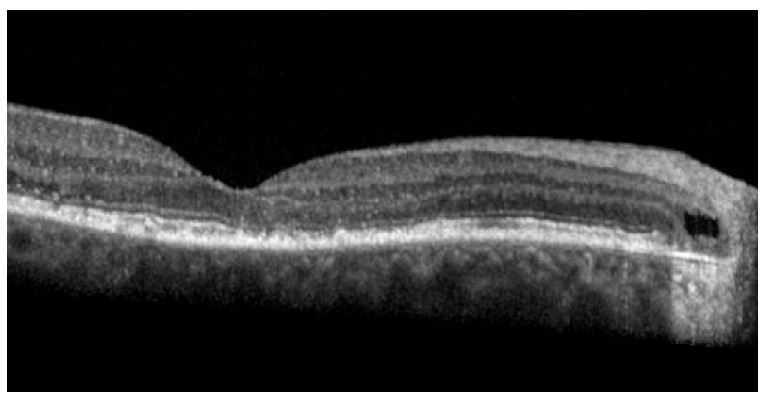

Figure 3 Beneath a cystic space in the outer peripapillary retina. Notes: The outer neurosensory retina and retinal pigment epithelium are attenuated, as seen on this magnified view of Figure IE.

A thickened choroid has been associated with serous retinal detachment. The choroid tends to be thick in eyes with central serous chorioretinopathy, nanophthalmos, and primary UES, all of which share a frequent common feature of hyperopia. ${ }^{1,3}$ Increased transudation of plasma from the thickened choroid is the presumed cause of the intraretinal and subretinal fluid. ${ }^{4}$ Margolis and Spaide ${ }^{5}$ reported the mean subfoveal choroidal thickness to be $287 \pm 26 \mu \mathrm{m}$. In the present case, the choroidal thickness was $496 \mu \mathrm{m}$ OD and $537 \mu \mathrm{m}$ OS. We propose that the thickened posterior choroid in this case may have resulted in hyperpermeability, as is suggested by late hyperfluorescence on indocyanine green angiography.

The increased fluid transudation may leave the choroid or be retained. Fluid in the choroid may leave the eye by

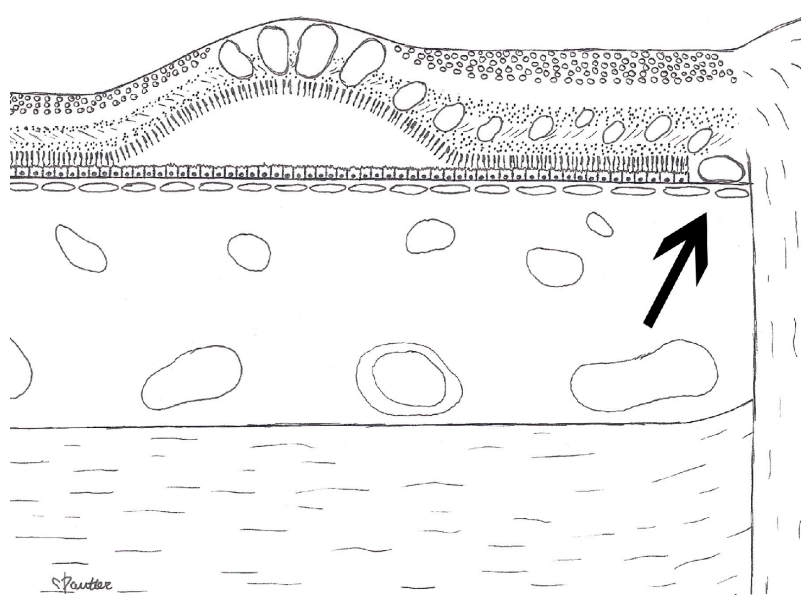

Figure 4 Artist rendition of the macula.

Notes: This artist rendition of the macula shows proposed site of fluid leakage from the choroid into the retina (arrow). Fluid passes from the choroid through Bruch's membrane at the temporal crescent, where the fluid passes directly into the retina because of the attenuation of the RPE and photoreceptor layers. The fluid gravitates toward the macula, where it passes through the external limiting membrane into the subfoveal neurosensory space.

Abbreviation: RPE, retinal pigment epithelium. diffusion through the sclera or through scleral emissaria. The resistance of fluid to diffuse through the sclera may be related to the thickness as well as to the histology of the sclera. ${ }^{6}$ If resistance is high, the fluid may collect in the suprachoroidal space and cause a choroidal detachment, as is seen in UES. ${ }^{1}$ Alternatively, the extravascular fluid in the choroid may enter the subretinal space. The site of entry into the subretinal space in UES is unknown, although the reason for entry is speculated to be a breakdown of the RPE blood-ocular barrier. ${ }^{1,7}$ In the present case, there was no suprachoroidal fluid detected and no breakdown of the RPE detected by fluorescein angiography. In posterior uveal effusion, the optic disc margin may be the site of entry of fluid from the choroid into the retina and subretinal space, as described in the following paragraph.

Predisposing factors for leakage from the optic disc margin include an abnormally thick peripapillary choroid and the presence of a disc crescent. In normal eyes, the posterior choroid is thickest beneath the fovea. ${ }^{5}$ In this case, the choroid was thickest between the fovea and the disc. The unique peripapillary disc anatomy at the temporal crescent may present a weak link in the blood-retinal barrier. ${ }^{8}{ }^{8}$ With the use of OCT, temporal crescents feature disruptions of the external limiting membrane at the temporal edge of the cresent. Intraretinal cystic spaces overlying the myopic temporal crescent have been demonstrated. ${ }^{10}$ Outer neurosensory tissue loss over the crescent may explain the reason that, instead of subretinal fluid, intraretinal fluid from the choroid is present. The OCT in our case demonstrated the attenuation of the RPE at the temporal margin of the crescent leaving Bruch's membrane as the only barrier between the thick choroid and the retina. Bruch's membrane only blocks passage of large molecules and allows passage of water. ${ }^{11,12}$ Indeed, the disc border is a common site of serous exudation in chronic central serous tilted disc syndrome ${ }^{13}$ and is described in immunogammopathies. ${ }^{14}$ In the present case, the choroid was thickest at the disc, and the leakage progressed from and receded to an epicenter at the disc margin, which was associated with a crescent that stained on fluorescein angiography. The combination of the unique location of choroidal thickness at the disc in this case and the presence of a crescent may have resulted in serous macular detachment without choroidal detachment, which appears to antedate the serous retinal detachment in primary UES. The leakier blood-ocular barrier at the disc may have provided an outlet from the effusion and thereby prevented suprachoroidal accumulation. Furthermore, resistance to suprachoroidal fluid in the posterior pole is provided by the 
attachment of numerous penetrating posterior ciliary arteries and by the firm attachment of the choroid and retina at the disc. This may explain the general absence of posterior choroidal detachment in the setting of peripheral choroidal detachment in UES. ${ }^{1}$

Similar to what is seen in primary UES, cataract extraction appears to have exacerbated the choroidal effusion in the present case. ${ }^{1}$ Possible mechanisms may include inflammation and ocular hypotension, both of which are seen with surgery. ${ }^{15}$ Although in this case there was no overt sign of unusual inflammation after cataract surgery, the severity of the effusion appeared to abate over time after surgery and, therefore, required a corresponding reduction in treatment.

In this case report, acetazolamide successfully resolved the macular edema and detachment. Carbonic anhydrase is present in the cell membrane of the RPE. ${ }^{16}$ Acetazolamide increases the RPE pump and has been used to treat cystoid macular edema and serous macular detachment. ${ }^{17}$ In this case, acetazolamide was effective orally for acute treatment and was used topically for maintenance. Topical therapy has been shown to have effects in the posterior pole. ${ }^{18}$ The authors are not aware of the use of carbonic anhydrase inhibitors in the treatment of serous retinal detachment in UES. In UES, carbonic anhydrase inhibitors may be expected to increase fluid transport from the subretinal space into the choroid and thereby promote peripheral choroidal detachment. ${ }^{19}$ In isolated posterior uveal effusion, the focal posterior location of choroidal thickening may explain the positive response to therapy. Indeed, carbonic anhydrase inhibitors may be of value only for the limited form of isolated posterior uveal effusion presented herein. Future use of photodynamic therapy may be considered to thin the posterior choroid in cases of failure of or lack of tolerance to medical therapy. ${ }^{20}$

\section{Conclusion}

In summary, we present a case of serous macular detachment and macular edema associated with hyperopia, focal posterior thickening of the choroid, and posterior uveal effusion. The effusion was exacerbated by cataract surgery and was treated successfully with carbonic anhydrase inhibitors. This case may represent an isolated posterior manifestation in the spectrum of clinical presentations of the UES. With the increasing use of swept-source OCT, a greater understanding of the prevalence and etiology of this condition is likely.

\section{Disclosure}

The authors report no conflicts of interest in this work.

\section{References}

1. Elagouz M, Stanescu-Segall D, Jackson TL. Uveal effusion syndrome. Surv Ophthalmol. 2010;55(2):134-145.

2. Harada T, Machida S, Fujiwara T, Nishida Y, Kurosaka D. Choroidal findings in idiopathic uveal effusion syndrome. Clin Ophthalmol. 2011;5:1599-1601.

3. Imamura Y, Fujiwara T, Margolis R, Spaide RF. Enhanced depth imaging optical coherence tomography of the choroid in central serous chorioretinopathy. Retina. 2009;29(10):1469-1473.

4. Cho HJ, Kim HS, Jang YS, et al. Effects of choroidal vascular hyperpermeability on anti-vascular endothelial growth factor treatment for polypoidal choroidal vasculopathy. Am J Ophthalmol. 2013;156(6):1192-1200.

5. Margolis R, Spaide RF. A pilot study of enhanced depth imaging optical coherence tomography of the choroid in normal eyes. Am J Ophthalmol. 2009;147(5):811-815.

6. Jackson TL, Hussain A, Salisbury J, Sherwood R, Sullivan PM, Marshall J. Transscleral albumin diffusion and suprachoroidal albumin concentration in uveal effusion syndrome. Retina. 2012;32(1):177-182.

7. Gass JD. Uveal effusion syndrome. A new hypothesis concerning pathogenesis and technique of surgical treatment. Retina. 1983; 3(3):159-163.

8. Chauhan BC, Burgoyne CF. From clinical examination of the optic disc to clinical assessment of the optic nerve head: a paradigm change. $\mathrm{Am}$ J Ophthalmol. 2013;156(2):218-227.

9. Laties AM, Rapoport S. The blood-ocular barriers under osmotic stress. Studies on the freeze-dried eye. Arch Ophthalmol. 1976;94(7): 1086-1091.

10. Chui TY, Zhong Z, Burns SA. The relationship between peripapillary crescent and axial length: Implications for differential eye growth. Vision Res. 2011;51(19):2132-2138.

11. Cunha-Vaz J. The blood-ocular barriers. Surv Ophthalmol. 1979;23(5):279-296.

12. Hussain AA, Starita C, Hodgetts A, Marshall J. Macromolecular diffusion characteristics of ageing human Bruch's membrane: implications for age-related macular degeneration (AMD). Exp Eye Res. 2010;90(6):703-710.

13. Nakanishi H, Tsujikawa A, Gotoh N, et al. Macular complications on the border of an inferior staphyloma associated with tilted disc syndrome. Retina. 2008;28(10):1493-1501.

14. Rusu IM, Mrejen S, Engelbert M, et al. Immunogammopathies and acquired vitelliform detachments: a report of four cases. Am J Ophthalmol. 2014;157(3):648-657.

15. Yalvac IS, Satana B, Ozkan G, Eksioglu U, Duman S. Management of glaucoma in patients with nanophthalmos. Eye (Lond). 2008;22(6):838-843.

16. Wolfensberger TJ, Mahieu I, Jarvis-Evans J, et al. Membrane-bound carbonic anhydrase in human retinal pigment epithelium. Invest Ophthalmol Vis Sci. 1994;35(9):3401-3407.

17. Wolfensberger TJ. The role of carbonic anhydrase inhibitors in the management of macular edema. Doc Ophthalmol. 1999;97(3-4): 387-397.

18. Siesky B, Harris A, Brizendine E, et al. Literature review and metaanalysis of topical carbonic anhydrase inhibitors and ocular blood flow. Surv Ophthalmol. 2009;54(1):33-46.

19. Lee GC, Tam CP, Danesh-Meyer HV, Myers JS, Katz LJ. Bilateral angle closure glaucoma induced by sulphonamide-derived medications. Clin Experiment Ophthalmol. 2007;35(1):55-58.

20. Sonoda S, Sakamoto T, Yamashita T, et al. Choroidal structure in normal eyes and after photodynamic therapy determined by binarization of optical coherence tomographic images. Invest Ophthalmol Vis Sci. 2014;55(6):3893-3899. 
Clinical Ophthalmology

\section{Publish your work in this journal}

Clinical Ophthalmology is an international, peer-reviewed journal covering all subspecialties within ophthalmology. Key topics include: Optometry; Visual science; Pharmacology and drug therapy in eye diseases; Basic Sciences; Primary and Secondary eye care; Patien Safety and Quality of Care Improvements. This journal is indexed on

PubMed Central and CAS, and is the official journal of The Society of Clinical Ophthalmology (SCO). The manuscript management system is completely online and includes a very quick and fair peer-review system, which is all easy to use. Visit http://www.dovepress.com/ testimonials.php to read real quotes from published authors. 